\title{
Efruxifermin in non-alcoholic steatohepatitis: a randomized, double-blind, placebo-controlled, phase 2 a trial
}

\author{
Stephen A. Harrison', Peter J. Ruane ${ }^{2}$, Bradley L. Freilich ${ }^{3}$, Guy Neff ${ }^{4}$, Rashmee Patil ${ }^{5}$, \\ Cynthia A. Behling ${ }^{6}$, Chen Hu${ }^{7}$, Erica Fong ${ }^{8}$, Brittany de Temple ${ }^{8}$, Erik J. Tillman ${ }^{8}{ }^{8}$, Timothy P. Rolph ${ }^{8}$, \\ Andrew Cheng ${ }^{8}$ and Kitty Yale ${ }^{8}{ }^{\mathrm{M}}$
}

Preclinical and clinical data suggest that fibroblast growth factor 21 (FGF21) is anti-fibrotic, improves metabolic status and has potential to treat non-alcoholic steatohepatitis (NASH). We assessed the safety and efficacy of efruxifermin, a long-acting FC-FGF21 fusion protein, for the treatment of NASH. BALANCED was a randomized, placebo-controlled study in patients with NASH conducted at 27 centers in the United States (ClinicalTrials.gov NCT03976401). Eighty patients, stratified by hepatic fat fraction (HFF) and fibrosis stage, were randomized using a centrally administered minimization algorithm 1:1:1:1 to receive placebo $(n=21)$ or efruxifermin $28 \mathrm{mg}(n=19)$, efruxifermin $50 \mathrm{mg}(n=20)$ or efruxifermin $70 \mathrm{mg}(n=20)$ via weekly subcutaneous injection for 16 weeks. The primary endpoint-absolute change from baseline in HFF measured as magnetic resonance imagingproton density fat fraction at week 12-was met. For the full analysis set, the least squares mean absolute changes (one-sided 97.5\% confidence interval) from baseline in HFF were $-12.3 \%$ (-infinity (-inf), -10.3$),-13.4 \%(-$ inf, -11.4$)$ and $-14.1 \%(-$ inf, -12.1) in the 28-, 50- and 70-mg groups, respectively, versus $0.3 \%$ (-inf, 1.6) in the placebo group, with statistically significant differences between efruxifermin groups and placebo $(P<0.0001$ each). Overall, 70 of 79 patients who received the study drug (89\%) experienced at least one treatment-emergent adverse event (TEAE), with the majority grade 1-2 (64 (81\%)), five (6\%) grade 3 and one grade 4 . The most commonly reported drug-related TEAEs were grade 1-2 gastrointestinal (36 (46\%)). Treatment with efruxifermin significantly reduced HFF in patients with F1-F3 stage NASH, with an acceptable safety profile.

$\mathrm{N}$ on-alcoholic fatty liver disease (NAFLD) is associated with obesity, metabolic syndrome, dyslipidemia and type 2 diabetes (T2D). NASH is the progressive form of NAFLD, defined as $\geq 5 \%$ hepatic steatosis with inflammation and hepatocyte injury (that is, ballooning), with or without fibrosis ${ }^{1}$. Despite a predicted global prevalence of $2-12 \%$ in adults ${ }^{2,3}$, currently there is no approved treatment. Most clinically evaluated therapeutic mechanisms target the metabolic dysfunction of hepatocytes or suppress inflammation and fibrosis ${ }^{4}$. However, an ideal therapeutic would treat advanced fibrosis and resolve the profibrotic milieu driven by hepatocyte death (apoptosis) associated with chronic steatosis, lipotoxicity and oxidative and endoplasmic reticulum stress.

FGF21 is a key regulator of whole-body and individual organ metabolism ${ }^{5}$. It activates a cell membrane co-receptor complex of $\beta$-klotho and one of its cognate FGF receptors (FGFRs), FGFR1c, FGFR2c or FGFR3c. FGF21 and its analogs improve metabolic status in preclinical models of obesity, diabetes and $\mathrm{NASH}^{6}$, suggesting promise as a therapeutic for NASH. However, many FGF21 analogs have not fulfilled the preclinical promise of FGF21 therapeutics, with disappointing translation into the clinic. Although substantial reductions in serum triglycerides have been seen in patients with T2D and obesity, effects on high-density lipoprotein (HDL) and low-density lipoprotein (LDL) cholesterol have been variable, with inconsistent effects on glycemic control and body weight ${ }^{7-9}$. Furthermore, in patients with NASH, liver fat reductions have been moderate $^{10}$. Two antibody-based therapeutics that act on the FGF21 co-receptor complex are also in development. Unlike analogs of the FGF21 polypeptide, BFKB8488A (Genentech) and MK3655 (Merck Sharp \& Dohme; formerly NGM313 (NGM Biopharmaceuticals)) activate only the FGFR1c/ $\beta$-klotho co-receptor complex. In patients with NAFLD, the highest adequately tolerated dose of BFKB8488A reduced HFF by $38 \%$ after 12 weeks ${ }^{11}$ but did not improve markers of glycemic control in patients with $\mathrm{T} 2 \mathrm{D}^{12}$. After a single dose, MK3655 improved glycemic control in obese non-diabetic participants with $37 \%$ relative reduction in liver fat $36 \mathrm{~d}$ after administration; however, significant weight gain was seen ${ }^{13}$.

Efruxifermin is a fusion protein of human $\mathrm{IgG}_{1} \mathrm{Fc}$ domain linked to a modified human FGF21 (Fc-FGF21) with balanced in vitro agonist potency at FGFR1c, FGFR2c and FGFR3 $c^{14}$. Efruxifermin is longer acting than most FGF21 analogs, with a 3-3.5-d half-life ${ }^{15}$. A study in patients with T2D showed improvements in lipoprotein profiles and glycemic control ${ }^{15}$, which supported investigation of efruxifermin in patients with NASH. This study aimed to test the safety and efficacy of weekly subcutaneous administration for 16 weeks in patients with NASH.

\section{Results}

Between 28 May and 15 November 2019, 356 individuals were screened at 27 sites, with 80 patients from 24 sites enrolled between 2 July and 16 December 2019 to receive efruxifermin $(n=59)$ or

Pinnacle Clinical Research, San Antonio, TX, USA. ${ }^{2}$ Ruane Clinical Research, Los Angeles, CA, USA. ${ }^{3}$ Kansas City Research Institute, Kansas City, MO, USA. ${ }^{4}$ Covenant Research, LLC, Sarasota, FL, USA. ${ }^{5}$ South Texas Research Institute, Edinburg, TX, USA. ${ }^{6}$ NAFLD Research Center, Division of Gastroenterology, University of California, San Diego, San Diego CA, USA. ${ }^{7}$ Medpace, Inc., Cincinnati, OH, USA. ${ }^{8}$ Akero Therapeutics, South San Francisco, CA, USA. 凶e-mail: kyale@akerotx.com 


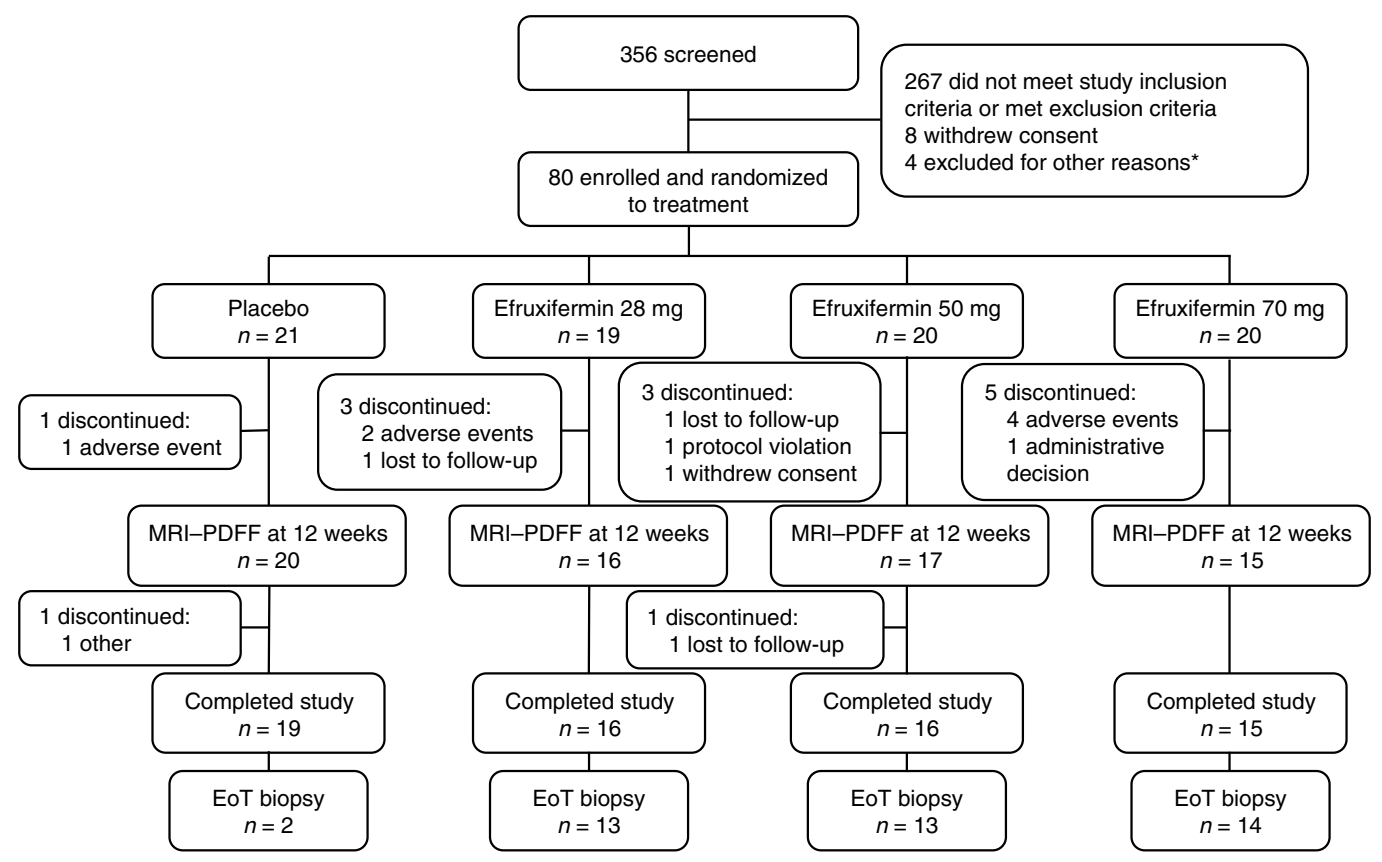

Fig. 1 | Clinical study flow diagram. EoT, end of treatment. "Might be excluded for more than one reason.

placebo $(n=21)$ (Fig. 1). The full analysis set (FAS), comprising all randomized participants, totaled 80 patients. Seventy-nine patients were included in the safety set, and one patient randomized to the 50-mg cohort was not dosed, owing to meeting exclusion criteria. The magnetic resonance imaging-proton density fat fraction (MRIPDFF) evaluable analysis set (MAS) included all FAS participants who had baseline and week 12 HFF assessed by MRI-PDFF and comprised 68 patients. The liver biopsy evaluable analysis set (BAS) included all FAS participants with baseline and end-of-treatment liver biopsy results and comprised 42 patients.

Baseline demographics and disease characteristics. Baseline demographics and disease characteristics were generally similar across treatment groups (Table 1). Mean patient weight (s.d.) was $103.5(21.9) \mathrm{kg}$, and 71/80 (89\%) randomized patients had a body mass index of $\geq 30 \mathrm{~kg} \mathrm{~m}^{-2}$. Fibrosis stages (F1 (36\%), F2 (33\%) or F3 (31\%)) were evenly distributed across treatment groups. Overall, mean HFF (s.d.) was $19.6 \%$ (6.9\%) and similar for all treatment groups; $56 / 80$ (70\%) patients had an HFF $\geq 15 \%$. Total cholesterol, non-HDL cholesterol and triglycerides were slightly higher in the placebo group but similar across efruxifermin groups. During the course of the study, $37 \%$ of patients were receiving statin therapy. Mean baseline glycated hemoglobin (HbAlc) values $(\geq 6.2 \%$ in all groups) suggest sub-optimal glycemic control among participants, despite $51 \%$ of patients receiving anti-diabetic medication. Mean (s.d.) endogenous FGF21 concentration at baseline was 529 (394) $\mathrm{ng} \mathrm{L}^{-1}$.

Change in HFF. The primary objective of the study-absolute reduction in $\mathrm{HFF}$ at week 12-was met. A significant reduction in HFF at week 12 was observed in all efruxifermin-treated groups (Fig. 2a). The least squares (LS) mean absolute changes (one-sided 97.5\% confidence interval (CI)) from baseline in HFF were $-12.3 \%$ ((-inf), -10.3$),-13.4 \%$ (-inf, -11.4$)$ and $-14.1 \%(-$ inf, -12.1$)$ in the 28-, 50- and 70-mg groups, respectively, and $0.3 \%$ (-inf, 1.6) in the placebo group (Fig. 2a), with significant differences between efruxifermin-treated groups and placebo $(P<0.0001$ for each).

The LS mean relative change in HFF (secondary outcome) from baseline to week 12 (two-sided 95\% CI) was $-63.2 \%(-73.0,-53.4$ ),
$-70.9 \%(-80.7,-61.2)$ and $-72.3 \%(-82.1,-62.4)$, respectively, for participants receiving $28 \mathrm{mg}, 50 \mathrm{mg}$ and $70 \mathrm{mg}$ and $-0.3 \%(-9.2$, 8.6) for placebo participants (Fig. 2b). The reductions were significantly greater than placebo for each efruxifermin group: $62.9 \%$, $70.6 \%$ and $72.0 \%$ in the $28-, 50-$ and $70-\mathrm{mg}$ groups, respectively $(P<0.0001$ for each). The LS mean relative reduction in HFF at week 6 was $83-91 \%$ of the reduction at 12 weeks, indicating a rapid effect (Supplementary Table 1). The magnitude of efruxifermin-induced HFF reduction was similar in F1 compared to F2-F3 patient subgroups at 12 weeks (Supplementary Table 2).

Responder analyses. The proportion of patients in the FAS with $\geq 30 \%$ relative reduction of HFF (secondary outcome) at week 12 (MRI-PDFF responders) was greater in all efruxifermin groups compared to the placebo group (Table 2). All efruxifermin-treated patients with HFF measurement at week 12 achieved $\geq 30 \%$ relative reduction and were, therefore, eligible for an end-of-treatment liver biopsy. In contrast, HFF was reduced by $\geq 30 \%$ in only two patients in the placebo group. Furthermore, two-thirds of patients receiving $28 \mathrm{mg}$, and almost all patients in the higher-dose groups, achieved $\geq 50 \%$ relative $\mathrm{HFF}$ reduction, whereas $60 \%$ of efruxifermin-treated patients achieved $\geq 70 \%$ reduction. The magnitude of these reductions resulted in $25 \%, 53 \%$ and $67 \%$ of patients in the $28-, 50$ - and 70 -mg groups, respectively, achieving normalization of liver fat $(\leq 5 \% \mathrm{HFF})$ by 12 weeks.

End-of-treatment biopsies were obtained from 2, 13, 13 and 14 HFF responders in the placebo, 28-, 50- and 70-mg groups, respectively (BAS). Biopsies were not obtained from eight eligible patients in efruxifermin-treated groups due to Coronavirus Disease 2019 (COVID-19) and/or patient-specific issues.

Markers of liver function. The change from baseline in levels of the liver injury marker alanine aminotransferase (ALT) at week 12 was a secondary outcome; all other liver enzyme endpoints were exploratory outcomes. In line with reduced HFF, ALT decreased with time in all efruxifermin groups and remained at week 8 levels throughout treatment (Fig. 3a). The LS mean absolute reduction from baseline at week 12 was significantly greater than placebo for all efruxifermin groups $(P=0.0004$ for $28 \mathrm{mg}$ and $P<0.0001$ for 50 - and $70-\mathrm{mg}$ 
Table 1 | Participant demographics and baseline characteristics

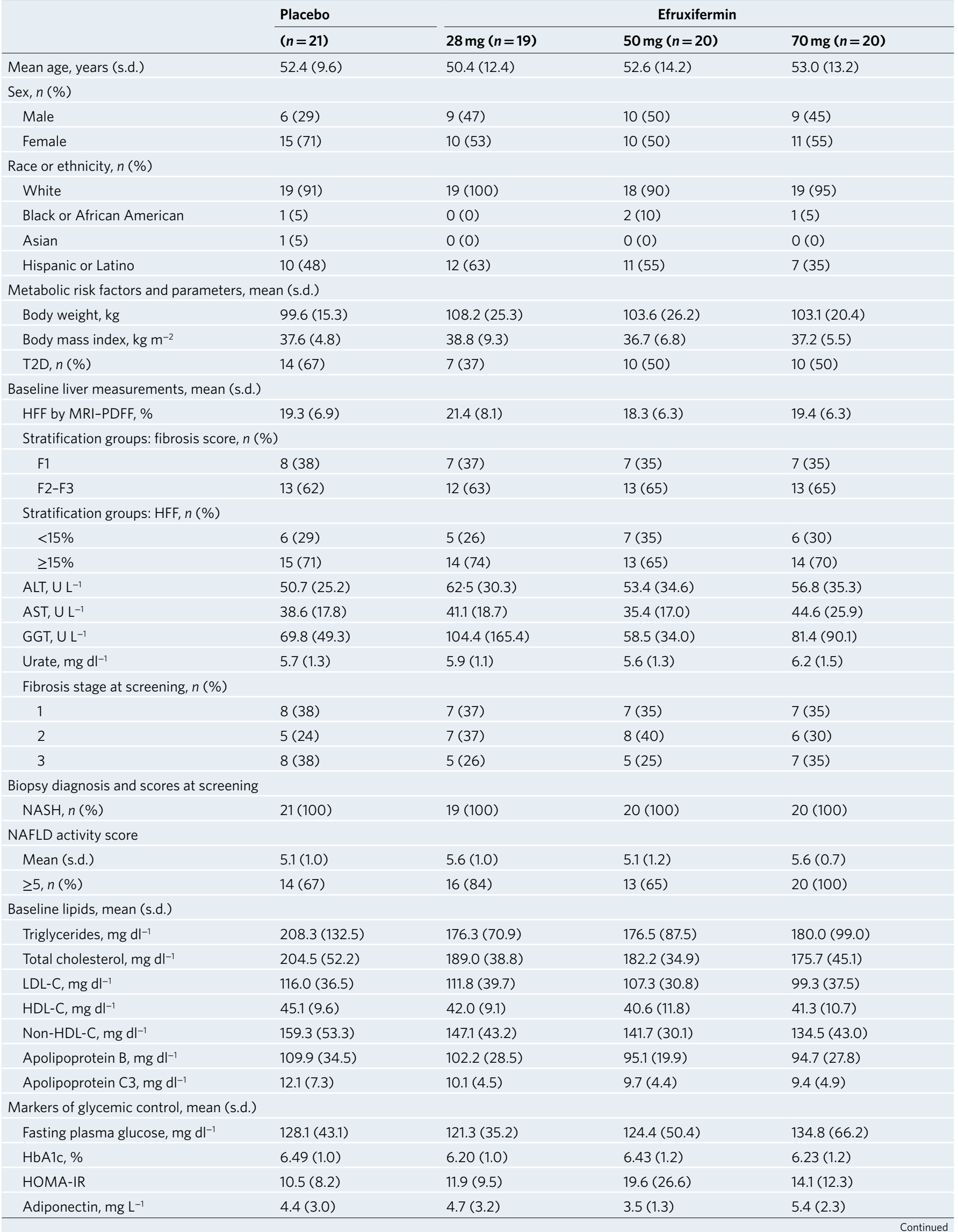


Table 1 | Participant demographics and baseline characteristics (continued)

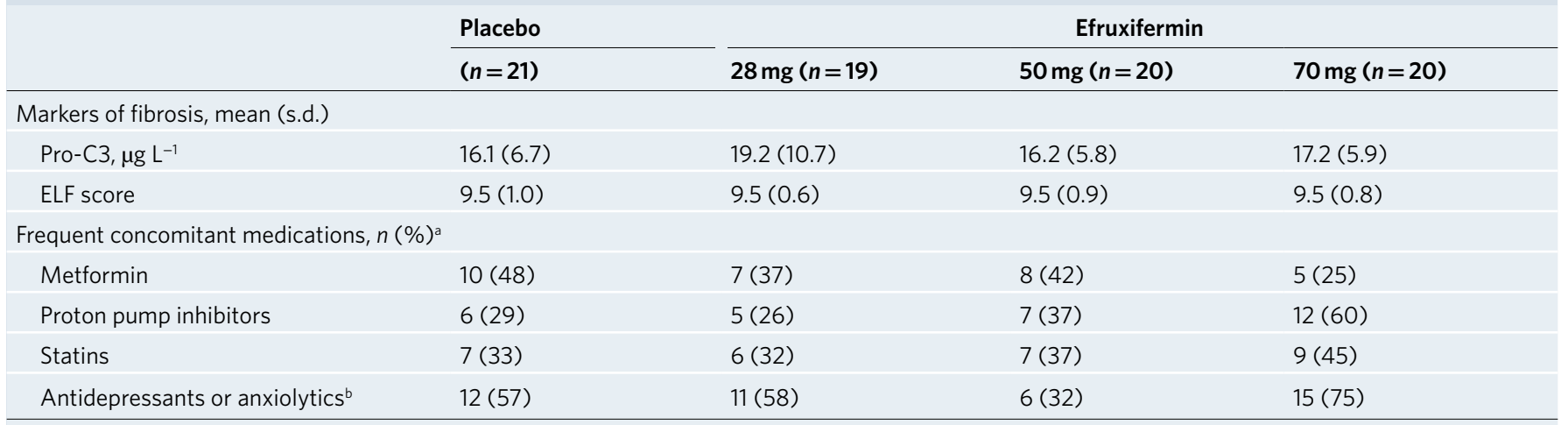

aThese values are for the safety set; all other baseline values are for the FAS. IIncludes the following World Health Organization Drug Dictionary Anatomical Therapeutic Chemical Classes: benzodiazepine derivatives; other antidepressants; and selective serotonin re-uptake inhibitors. HbA1c, hemoglobin A1c; HLD-C, high-density lipoprotein cholesterol; LDL-C, low-density lipoprotein cholesterol.
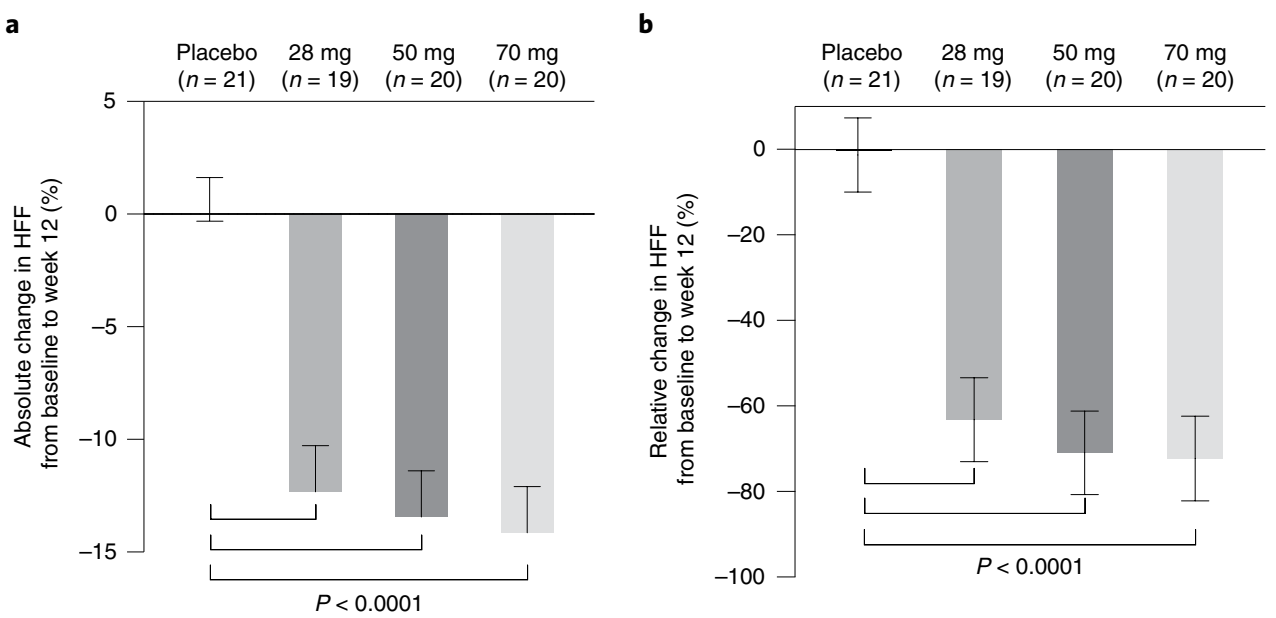

Fig. 2 | Absolute and relative change from baseline in HFF assessed by MRI-PDFF at week 12 (FAS). All efruxifermin treatment groups were significantly different from placebo $(P<0.0001$ for all) for both absolute and relative change. Columns represent LS means with error bars reflecting one-sided $97.5 \%$ Cls (a) or two-sided $95 \% \mathrm{Cls}(\mathbf{b})$ from an ANCOVA model with absolute (a) or relative (b) change from baseline as the dependent variable, treatment group and fibrosis score (F1 versus F2-F3) as factors and baseline value as a covariate. For participants in the analysis set with missing primary efficacy endpoints, multiple imputation by the fully conditional specification method was used.

groups (see Supplementary Table 3 for $P$ values at all measured time points for liver injury markers). Over half of the 28 -mg group and approximately two-thirds of patients in the 50- and 70-mg groups had $\geq 17 \mathrm{U} \mathrm{L}^{-1}$ decrease in ALT at week 16 compared to $11 \%$ of the placebo group (Table 2). Four weeks after treatment ceased, ALT levels had not increased in the 70-mg group. Although the 28- and 50 -mg groups returned toward baseline values, they remained significantly lower than placebo at 4 weeks after the last dose $(P=0.006$ and $P=0.005$, respectively). Of the other markers of liver injury, aspartate aminotransferase (AST) showed a similar magnitude and time course of reduction to ALT (Fig. 2b). AST levels were significantly lower than placebo at week 16 for all efruxifermin groups $(P=0.001, P=0.0003$ and $P<0.0001$ for $28 \mathrm{mg}, 50 \mathrm{mg}$ and $70 \mathrm{mg}$, respectively). Gamma glutamyl transferase (GGT) levels were more reduced in the 70-mg group than the $50-\mathrm{mg}$ and $28-\mathrm{mg}$ groups (Fig. 3c), with significant reductions from placebo for $50 \mathrm{mg}$ and $70 \mathrm{mg}$ at week $16(P=0.02$ and $P=0.0004$, respectively $)$. Alkaline phosphatase (ALP) levels were lower in efruxifermin-treated groups relative to placebo, although there was not a clear dose response (Fig. 3d; $P=0.008, P=0.02$ and $P=0.0003$ for $28-, 50$ - and $70-\mathrm{mg}$ groups at week 16). One patient receiving $28-\mathrm{mg}$ efruxifermin had an ALT spike $\left(659 \mathrm{U} \mathrm{L}^{-1}\right)$ at week 4 . The patient denied any intake of alcohol. Monitoring of drug-induced liver injury was initiated. When retested $4 \mathrm{~d}$ later, ALT levels had dropped substantially $\left(203 \mathrm{U} \mathrm{L}^{-1}\right)$. A transient increase at week 4 was also observed in AST and GGT in this patient, who did not meet criteria for drug-induced liver injury. Levels of urate, another marker of liver stress, were reduced by approximately $14-19 \%$ relative to placebo at week 16 (Fig. 3e; $P=0.0001, P<0.0001$ and $P=0.001$ for $28 \mathrm{mg}, 50 \mathrm{mg}$ and $70 \mathrm{mg}$, respectively, compared to placebo).

Consistent with reduced HFF and less liver injury, $78 \%$ of efruxifermin-treated patients in the BAS were NAFLD activity score (NAS) responders ( $\geq 2$ points without worsening of fibrosis-secondary outcome) (Table 2 ), and $48 \%$ had NASH resolution (0-1-point inflammation and 0-point ballooning components of NAS-exploratory outcome) without worsening of fibrosis. Markers of collagen synthesis and fibrogenesis were also reduced (Fig. 3g,f). At week 16, levels of N-terminal type III collagen pro-peptide (pro-C3) were significantly lower for all efruxifermin groups compared to placebo $(P=0.0002, P=0.004$ and $P=0.0002$ for 28-, 50- and 70-mg groups, respectively). Similarly, the enhanced liver fibrosis (ELF) scores at week 12 for efruxifermin groups were significantly less compared to placebo $(P=0.0008, P=0.0005$ and $P=0.03$ for $28 \mathrm{mg}, 50 \mathrm{mg}$ and $70 \mathrm{mg}$, respectively). The reduction 
Table 2 | Responder analyses

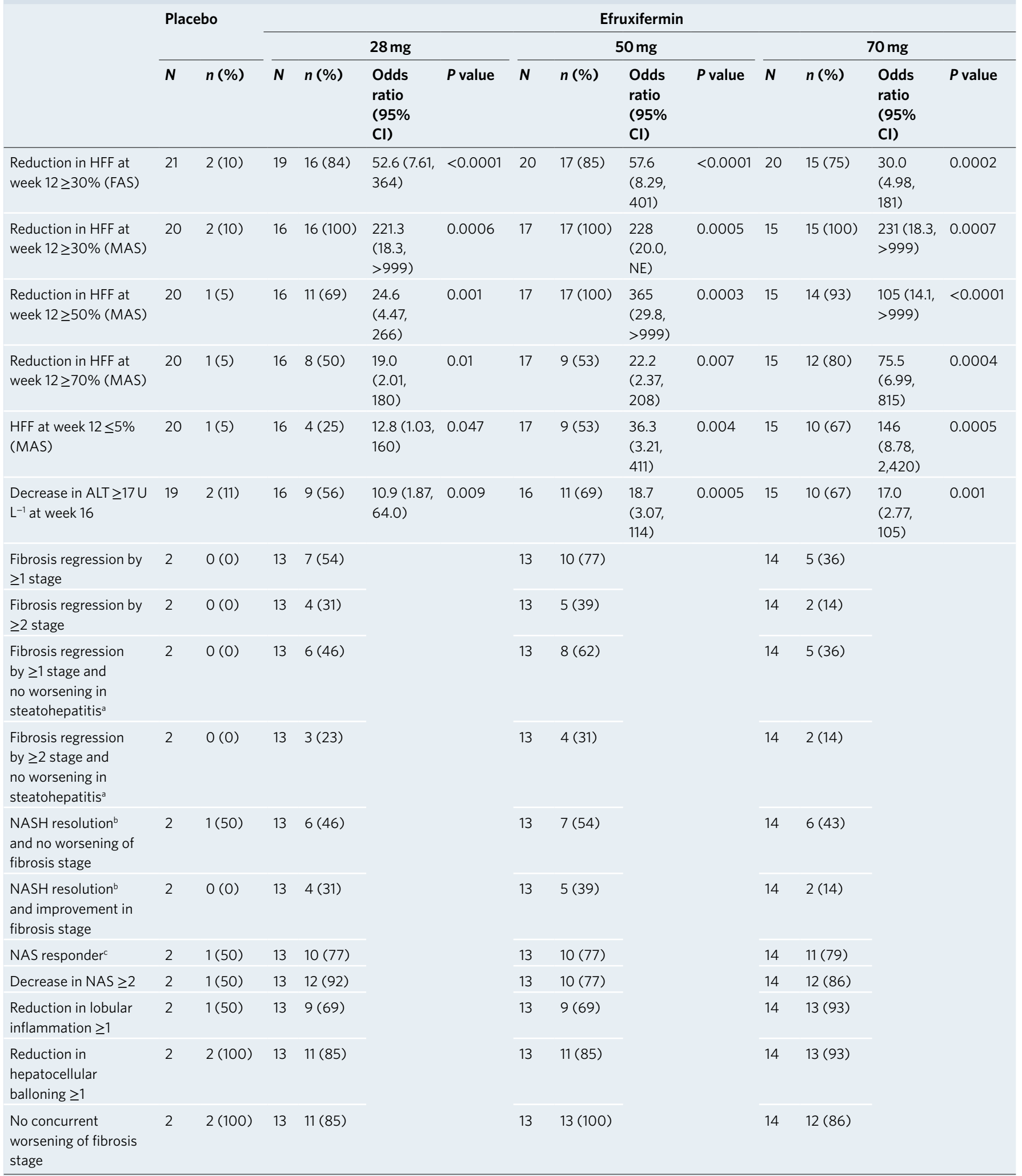

${ }^{\mathrm{a}} \mathrm{No}$ increase in NAS for ballooning, inflammation or steatosis ${ }^{\mathrm{b}} \mathrm{0}-1$ point inflammation and 0-point ballooning $\mathrm{NAS}$ improvement $\geq 2$ points (at least 1-point improvement in inflammation or ballooning) and no worsening of fibrosis. BAS, MRI-PDFF responders with liver biopsy at baseline and post-treatment analysis set; NAS, non-alcoholic fatty liver disease activity score; NE, not estimable. The proportions of patients in the FAS with $\geq 30 \%$ reduction in HFF at week 12 relative to baseline are presented, with last observation carried forward for any missing data. The proportions of patients in the MAS with $\geq 30 \%$ reduction, $\geq 50 \%$ reduction and $\geq 70 \%$ reduction and normalization of liver fat ( $\leq 5 \% \mathrm{HFF}$ ) are observed data. The proportions of patients with $\geq 17 \mathrm{U} \mathrm{L}^{-1}$ decrease in $\mathrm{ALT}$ are presented for participants with both baseline and week 16 values. These analyses and the proportion of patients in the BAS with improvements from baseline in histology measures were pre-specified in the statistical analysis plan. As only two patients receiving placebo were included in the BAS, a meaningful comparison with the placebo is not possible. For HFF reduction responder analyses, the odds ratios, corresponding two-sided $95 \% \mathrm{Cls}$ and $\mathrm{P}$ values were based on logistic regression with responder status as the dependent variable, treatment group and fibrosis score (F1 versus F2-F3) as factors and baseline HFF as a covariate. 
in systemic markers of fibrosis and fibrogenesis was associated with histological evidence of fibrosis regression in liver. Across all efruxifermin groups in the BAS, 55\% (22/40) had a fibrosis improvement of $\geq 1$ stage, and $28 \%(11 / 40)$ had $a \geq 2$ stage fibrosis improvement. Forty-eight percent (19/40) had a fibrosis improvement of $\geq 1$ stage without worsening of steatohepatitis, and $28 \%(11 / 40)$ had $\geq 1$ stage fibrosis improvement and resolution of NASH (all exploratory endpoints). As only two patients receiving placebo were included in the BAS, a meaningful comparison with placebo is not possible.

Safety. Overall, 70/79 (89\%) participants reported at least one TEAE; most were grade 1-2. Table 3 lists all TEAEs reported by $\geq 15 \%$ of the study population. There were no deaths. The most commonly reported drug-related adverse events (AEs) were gastrointestinal $(38 / 79(48 \%))$, mainly mild or moderate and transient.

Three drug-related AEs were considered grade 3: two events of diarrhea and one event of headache. One serious adverse event (SAE) of acute pancreatitis with subsequent diabetic ketoacidosis (grade 4) was considered related to the study drug and led to discontinuation. This patient was morbidly obese with severe insulin resistance before the study start, as indicated by acanthosis nigricans and hyperinsulinemia. One patient experienced an SAE of abdominal pain (grade 2) and pyrexia (grade 1) related to the liver biopsy procedure at baseline. In total, seven patients discontinued due to TEAEs. None was due to the most common AE (diarrhea), and none was in the 50-mg group. One patient receiving $70 \mathrm{mg}$ discontinued due to dysphagia, which was considered unrelated to the study medication. Overall, 14/79 (18\%) participants experienced a procedure-related TEAE, all of which were grade 1 .

No clinically significant safety findings were identified based on laboratory parameters, blood pressure, heart rate, electrocardiograms or markers of bone turnover. Bone mineral density and salivary cortisol were unchanged from baseline to week 16 in all cohorts (Supplementary Table 4).

In participants who received at least one dose of efruxifermin, 41 of 57 evaluable individuals (72\%) were positive for anti-drug antibodies (ADAs) (Table 3). ADAs were first detected at week 8, with the highest prevalence at week 20 . The median titer at week 20 remained low at 1:8.7. Most ADA-positive samples were efruxifermin specific, although four ( $10 \%$ of ADA positive) participants' samples were cross-reactive to endogenous FGF21. All ADA-positive samples were non-neutralizing, except for one participant at week 20. A follow-up sample from this participant collected 43 weeks after the last dose was non-neutralizing. Consistent with a generally low titer ADA response, serum markers of the biological actions of efruxifermin were maintained over 16 weeks of treatment.

Markers of lipid metabolism and glycemic control. In addition to evidence of better liver health, markers of lipid metabolism and glycemic control (exploratory outcomes) indicate improved whole-body metabolic homeostasis (Supplementary Table 5). Lipoprotein profiles were improved by a similar magnitude for all efruxifermin groups. Total cholesterol levels were significantly lower $(-8 \%)$ at $28 \mathrm{mg}$ and numerically lower $(-4 \%)$ for $50 \mathrm{mg}$ and
$70 \mathrm{mg}$ compared to placebo at week 16. The percent change from baseline to week 16 for HDL cholesterol and non-HDL cholesterol across efruxifermin groups compared to placebo was $+33 \%$ to $+38 \%$ and $-15 \%$ to $-20 \%$, respectively. Consistent with the decrease in non-HDL cholesterol, apolipoprotein B levels were reduced significantly by $12-16 \%$ at week 16 compared to placebo for $28-, 50$ and 70-mg groups. LDL cholesterol levels at week 16 were reduced by $16 \%$ in the 28 -mg group but were relatively unchanged in the $50-\mathrm{mg}(-4 \%)$ and $70-\mathrm{mg}(-7 \%)$ groups compared to placebo. The dose-related pattern ranging from reduction to minimal change in LDL cholesterol for the subset of patients taking statin medication was similar to those who were not, and likewise for those with baseline LDL cholesterol $\geq 100 \mathrm{mg} \mathrm{dl}^{-1}$ versus $\leq 100 \mathrm{mg} \mathrm{dl}^{-1}$. The reduction in triglycerides from baseline was $51-61 \%$ across efruxifermin groups compared to placebo. Consistent with this, apolipoprotein C3 levels declined to a similar extent compared to placebo $(-27 \%$ to $-29 \%$ ) across all efruxifermin groups.

Higher efruxifermin doses generally showed greater improvements in glycemic control. Placebo-adjusted LS mean reductions in $\mathrm{HbA} 1 \mathrm{c}$ at week 16 were significant in the 50 - and 70 -mg groups, with changes in fasting glucose levels in line with Hb1Ac reductions (Supplementary Table 5). Improved glycemic control was associated with enhanced insulin sensitivity; LS mean homeostasis model assessment of insulin resistance (HOMA-IR) values were significantly less than placebo for the 70-mg group. Consistent with greater insulin sensitivity, LS mean C-peptide levels were significantly lower at all doses compared to placebo. Insulin LS mean levels were also significantly lower for the 70-mg dose and numerically lower for $28 \mathrm{mg}$ and $50 \mathrm{mg}$. A post hoc analysis of FAS patients with T2D $(n=41)$ suggests that efruxifermin-associated improvements in glycemic control were greater in those with T2D $(n=39)$ than the overall FAS. Notably, there were numerically greater LS mean reductions versus placebo in $\mathrm{HbAlc}$ in the $50-\mathrm{mg}(0.6 \%)$ and $70-\mathrm{mg}$ (1.0\%) dose groups in the T2D subgroup (Supplementary Table 6) compared to the FAS $50-\mathrm{mg}(0.5 \%)$ and $70-\mathrm{mg}(0.6 \%)$ dose groups (Supplementary Table 5). The greater decrease in participants with $\mathrm{T} 2 \mathrm{D}$ is in keeping with higher baseline $\mathrm{HbA1c}$ in that subgroup (6.9-7.0\%) compared to the subgroup without a diagnosis of T2D (5.5-5.8\%).

Of note, body weight was significantly reduced by $3.3 \mathrm{~kg}$ relative to baseline compared to placebo in the $70-\mathrm{mg}$ group. A numerical, non-significant mean reduction of $2.2 \mathrm{~kg}$ was seen in the 50-mg group (Supplementary Table 5). The lower body weight was associated with a statistically significant increase in total body fat (Supplementary Table 5).

\section{Discussion}

Treatment with efruxifermin for 12 weeks was associated with a reduction of absolute HFF ranging from $12 \%$ to $14 \%$ across all doses. Within the MAS, all efruxifermin-treated patients achieved $\geq 30 \%$, and $88 \%$ achieved $\geq 50 \%$, relative reduction in liver fat, whereas HFF was normalized in $67 \%$ of patients at the highest dose (70 mg). Two sources account for $\sim 80 \%$ of the flux of fatty acids in the liver of patients with NAFLD: free fatty acids released by adipose

Fig. 3 | Markers of liver injury. Time courses for change from baseline in levels of ALT (a), AST (b), GGT (c), ALP (d), urate (e) and mean levels of pro-C3 (f) and ELF score (g). AST, GGT, ALP and urate data are LS mean $\pm 95 \% \mathrm{Cl}$ from a post hoc mixed-model repeated-measures analysis, with absolute change from baseline as the dependent variable; treatment group, fibrosis score (F1 versus F2-3), HFF (<15\% versus $\geq 15 \%$ ) and visit as factors; and baseline value as a covariate, as well as interaction of treatment group by visit. Only participants with non-missing baseline and the specified visit are included. There were no adjustments for multiple comparisons. This analysis was pre-planned for ALT. The two-sided $P$ values for comparison with placebo from these analyses at all tested data points and number of participants in each group at each time point are provided in Supplementary Table 3. Pro-C3 levels and ELF score data are plotted as mean \pm s.d., with the last observation carried forward for any missing data. The two-sided $P$ values for comparison with placebo at all tested data points for ELF score and pro-C3 come from an ANCOVA model, with absolute change from baseline as the dependent variable; treatment group, fibrosis score (F1 versus F2-3) and HFF ( $<15 \%$ versus $\geq 15 \%$ ) as factors; and baseline value as a covariate, and are provided in Supplementary Table 3. 
tissue lipolysis $(\sim 50 \%)$ and de novo lipogenesis (DNL) in the liver $(\sim 30 \%)^{16}$. Based on tissue-specific expression of FGF21's receptors ${ }^{17}$, FGF21-induced inhibition of adipose tissue lipolysis is mediated by
FGFR1c, and direct suppression of DNL in liver ${ }^{18}$ is mediated by FGFR2c, the most highly expressed FGF21 receptor, and possibly by FGFR3c. FGFR1c agonists BFKB8488 and MK3655 (NGM313),
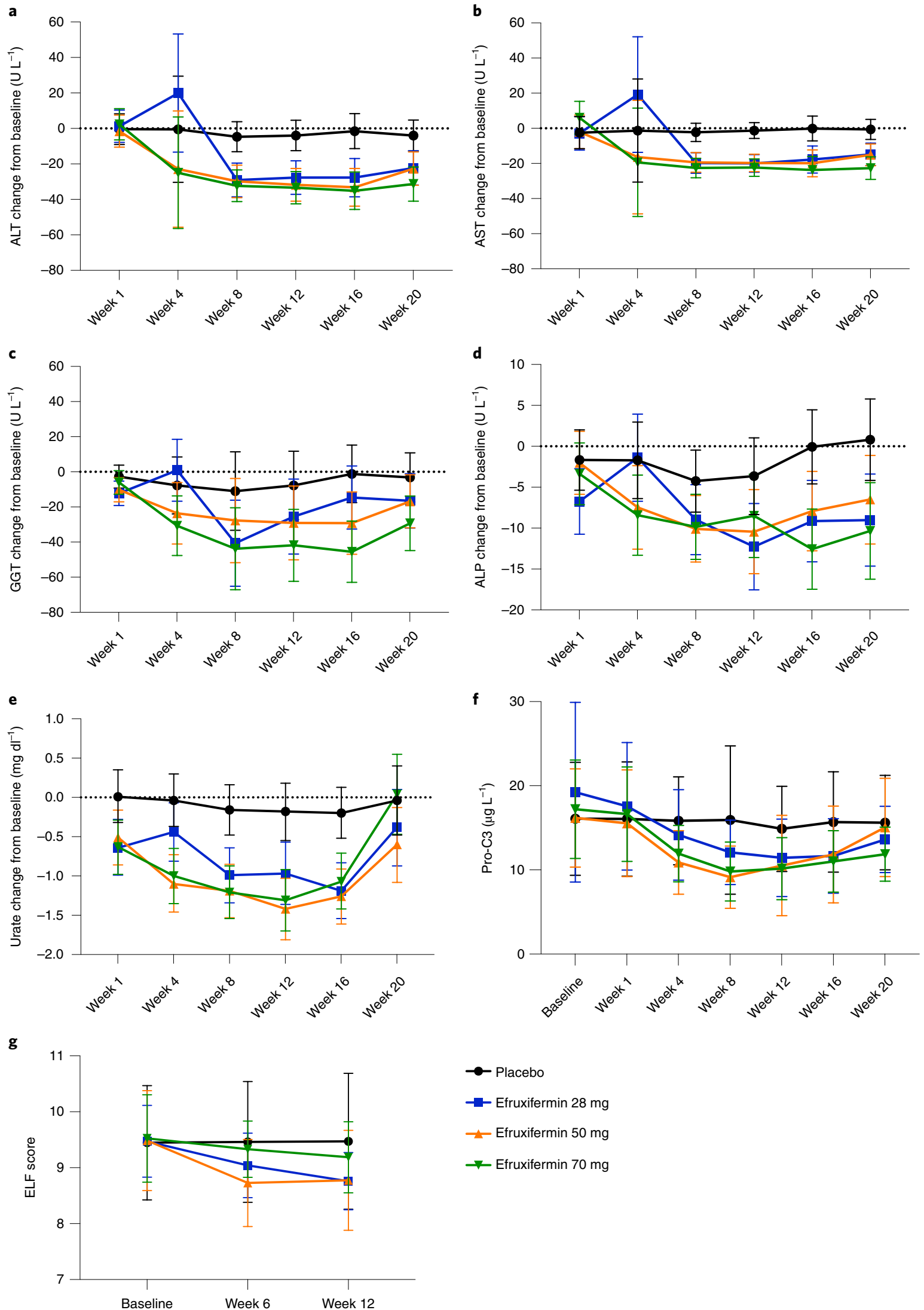

Placebo

- Efruxifermin $28 \mathrm{mg}$

$\rightarrow$ Efruxifermin $50 \mathrm{mg}$

$\rightarrow$ Efruxifermin $70 \mathrm{mg}$ 
Table 3 | Adverse events

\begin{tabular}{|c|c|c|c|c|}
\hline & \multirow{2}{*}{$\begin{array}{l}\text { Placebo } \\
n=21\end{array}$} & \multicolumn{3}{|c|}{ Efruxifermin } \\
\hline & & $\begin{array}{l}28 \mathrm{mg} \\
n=19\end{array}$ & $\begin{array}{l}50 \mathrm{mg} \\
n=19\end{array}$ & $\begin{array}{l}70 \mathrm{mg} \\
n=20\end{array}$ \\
\hline $\begin{array}{l}\text { Treatment-emergent } \\
\text { events, } n(\%)\end{array}$ & $16(76)$ & $18(95)$ & $17(90)$ & 19 (95) \\
\hline Life-threatening & $0(0)$ & $0(0)$ & $0(0)$ & $1(5)$ \\
\hline Severe & $1(5)$ & $1(5)$ & $2(11)$ & $1(5)$ \\
\hline Moderate & $8(38)$ & $11(58)$ & $12(63)$ & $15(75)$ \\
\hline Mild & $7(33)$ & $6(32)$ & $3(16)$ & $2(10)$ \\
\hline Study procedure related & $4(19)$ & $3(16)$ & $3(16)$ & $4(20)$ \\
\hline $\begin{array}{l}\text { Treatment-emergent } \\
\text { events leading to } \\
\text { discontinuation, } n(\%)\end{array}$ & $1(5)^{a}$ & $2(11)^{b}$ & $0(0)$ & $4(20)^{c}$ \\
\hline $\begin{array}{l}\text { Drug-related } \\
\text { treatment-emergent } \\
\text { events, } n(\%)\end{array}$ & $7(33)$ & $13(68)$ & $15(79)$ & $17(85)$ \\
\hline $\begin{array}{l}\text { Serious adverse events, } \\
n(\%)\end{array}$ & $0(0)$ & $1(5)^{d}$ & $0(0)$ & $1(5)$ \\
\hline \multicolumn{5}{|c|}{ Treatment-emergent events occuring in $\geq 15 \%, n(\%)$} \\
\hline Diarrhea & $4(19)$ & $5(26)$ & $11(58)$ & $7(35)$ \\
\hline Nausea & $1(5)$ & $8(42)$ & $5(26)$ & $12(60)$ \\
\hline Vomiting & $0(0)$ & $6(32)$ & $4(21)$ & $5(25)$ \\
\hline Abdominal pain & $1(5)$ & $1(5)$ & $3(16)$ & $4(20)$ \\
\hline $\begin{array}{l}\text { Frequent bowel } \\
\text { movements }\end{array}$ & $0(0)$ & $3(16)$ & $2(11)$ & $4(20)$ \\
\hline Fatigue & $4(19)$ & $1(5)$ & $1(5)$ & $7(35)$ \\
\hline Injection site erythema & $0(0)$ & $2(11)$ & $1(5)$ & $5(25)$ \\
\hline Injection site reaction & $1(5)$ & $2(11)$ & $1(5)$ & $4(20)$ \\
\hline Increased appetite & $1(5)$ & $4(21)$ & $5(26)$ & $5(25)$ \\
\hline Nasopharyngitis & $1(5)$ & $5(26)$ & $2(11)$ & $3(15)$ \\
\hline $\begin{array}{l}\text { Upper respiratory tract } \\
\text { infection }\end{array}$ & $2(10)$ & $2(11)$ & $1(5)$ & $3(15)$ \\
\hline ADAs, $n(\%)$ & $0(0)$ & $16(84)$ & $10(56)^{e}$ & $15(75)$ \\
\hline
\end{tabular}

aMuscular weakness and myalgia ${ }^{b}$ Nausea, vomiting and dysgeusia (one participant); panic attack, anxiety and tremor (one participant) 'Dysphagia (not drug related, one participant); acute pancreatitis (also an SAE, one participant); vomiting (one participant); and fatigue and nausea (one participant) ${ }^{d}$ Related to pre-dose liver biopsy ${ }^{e} n=18$

which mainly target adipose tissue, reduce HFF by $6-15 \%$ absolute after $5-12$ weeks of treatment ${ }^{11,13}$. The higher end of this range is similar to the magnitude of efruxifermin-elicited reduction, which, because of its balanced activity across FGFR $1 c / 2 c / 3 c^{14}$, has the potential to inhibit not only adipose tissue lipolysis but also liver DNL. Another FGF21 analog, pegbelfermin, reduced absolute HFF after 16 weeks of treatment by $\sim 6 \%{ }^{10}$. Pegbelfermin appears to have limited activity as an agonist of adipose tissue FGFR1c in humans, as evidenced by modest induction of adiponectin, a marker of FGFR1c activation, compared to efruxifermin and the FGFR1c-specific agonists $^{12,13}$. Consequently, it can be hypothesized that pegbelfermin acts predominantly on the liver receptors FGFR2c and FGFR3c, limiting its effects primarily to inhibition of DNL.

The FGF19 analog NGM282 (aldafermin) is an FGFR1c and FGFR4 agonist ${ }^{19}$ that reduced absolute HFF by $9-11 \%{ }^{20}$. Efruxifermin is not an FGFR4 agonist and reduced HFF by up to $14 \%$, calling into question the contribution of FGFR4 to the reduction of liver fat.
The correlation between the extent of reduction in HFF and resolution of NASH pathology has been evaluated by retrospective analyses of a small number of interventional study datasets. A relative reduction in HFF of $29 \%$ correlated with a $\geq 2$-point reduction in NAS ${ }^{21}$. Therefore, to avoid unnecessary liver biopsies in this study, only patients with $\geq 30 \%$ reduction in HFF were deemed eligible for end-of-treatment biopsy. Across all efruxifermin-treated patients with end-of-treatment biopsies, $85 \%$ had a $\geq 2$-point reduction in NAS, and $78 \%$ had $a \geq 2$-point reduction in NAS without worsening of fibrosis. This compares favorably with 12 weeks of open-label treatment with aldafermin ( $1 \mathrm{mg}$ and $3 \mathrm{mg}$ ), which was associated with a relative reduction of $58-67 \%$ in HFF and with $\mathrm{a} \geq 2$-point reduction in NAS without fibrosis worsening in $50-63 \%$ of patients ${ }^{22}$.

A higher threshold of $\geq 50 \%$ relative reduction in HFF appears to correlate with substantial resolution of NASH - that is, absence of ballooning and no or minimal lobular inflammation. Analysis of a 36-week study with resmetirom demonstrated that a reduction of $\geq 50 \%$ after 12 weeks of treatment was associated with $64 \%$ of patients achieving NASH resolution at the end of treatment (https:// ir.madrigalpharma.com/node/13331/pdf). Thus, substantial resolution of NASH histopathology might require a reduction in HFF of $\geq 50 \%$ rather than $\geq 30 \%$. A retrospective analysis from two NASH trials found that fibrosis reduction is most strongly predicted by NASH resolution ${ }^{23}$. Consistent with this, fibrosis was reduced in $61 \%$ of resmetirom-treated patients who achieved NASH resolution (https://ir.madrigalpharma.com/node/13331/pdf). Although efruxifermin was administered for only 16 weeks, $48 \%$ of treated patients meeting the $\geq 30 \%$ HFF threshold had $\geq 1$ stage improvement in fibrosis without NASH worsening, whereas $28 \%$ had both NASH resolution and fibrosis improvement. Qualitative histological analysis of paired biopsy samples suggested regression of fibrosis. The association of efruxifermin's reductions in HFF and NASH resolution with regression of fibrosis after 16 weeks of treatment strengthens the evidence that substantial reductions in HFF correlate with regression of NASH.

The reduction in HFF was accompanied by rapid, marked decreases in markers of liver stress and injury. Decreased ALT persisted for 4 weeks after efruxifermin treatment ceased, especially in the highest-dose group, suggesting sustained reduction in hepatocyte stress. Other markers of liver injury, including AST, GGT and ALP, were also substantially and consistently reduced. Serum urate, another marker associated with liver injury and elevated levels of tissue oxidative stress ${ }^{24}$, also declined by up to $20 \%$ after efruxifermin treatment. Urate levels have been associated with elevated ALT and GGT and increased risk of progression to liver cirrhosis and related hospitalization or death, even after adjustment for causes of, or risk factors for chronic liver disease $\mathrm{e}^{25}$.

Levels of the fibrogenesis marker pro-C3 are associated with extent of liver fibrosis ${ }^{26}$, and decreases in pro-C3 correlate with biopsy-proven improvements in hepatic fibrosis ${ }^{22}$. A substantial reduction of pro-C3 levels within 4 weeks of initiating efruxifermin treatment suggests that collagen synthesis is quickly suppressed. The rapid, parallel declines in HFF, markers of liver stress and fibrogenesis, might be attributable to a combination of efruxifermin's indirect and direct effects on fibrosis. By reducing HFF and hepatocyte stress, efruxifermin indirectly inhibits profibrotic signaling. Efruxifermin might also inhibit myofibroblast differentiation from hepatic stellate cells directly ${ }^{5}$ and indirectly via adiponectin ${ }^{27}$. Another biomarker of liver fibrosis is the ELF score, derived from a panel of biomarkers associated with matrix turnover. In longitudinal studies, a single unit increase of ELF score has been associated with a 2.53 -fold higher risk of a liver-related event ${ }^{28}$. The reduction of approximately 0.75 units of ELF score suggests that longterm efruxifermin treatment might result in lower risk of liver-related events. 
Diabetes, poor glycemic control and dyslipidemia are major contributors to cardiovascular-related mortality and morbidity in patients with $\mathrm{NASH}^{3}$. Exploratory analyses revealed that efruxifermin improved these common comorbidities sufficiently to restore a normal lipoprotein profile. In agreement with findings for the FGF21 analog LY2405319 ${ }^{7}$ and an earlier study with efruxifermin ${ }^{15}$, levels of apolipoprotein B were reduced by efruxifermin, consistent with lower levels of very low density lipoprotein secretion by liver ${ }^{29}$. Levels of apolipoprotein C3 were also reduced, which likely contributed to the reduced circulating triglyceride levels by increasing adipose tissue uptake via the action of lipoprotein lipase ${ }^{29}$. Notably, neither total nor LDL cholesterol were increased, in contrast to aldafermin treatment, which activates FGFR4 and suppresses expression of CYP7A1, thereby reducing flux of cholesterol to bile acid, in turn reducing clearance of LDL cholesterol ${ }^{20}$. Overall, the amelioration of dyslipidemia suggests that long-term treatment with efruxifermin might help to address the high prevalence of cardiovascular disease in patients with $\mathrm{NASH}^{1}$. Although FGF21 analogs generally improve dyslipidemia ${ }^{7-9,15}$, mostly they have not enhanced glycemic control ${ }^{7,8,12}$. In contrast, efruxifermin, like the long-acting FGFR1c agonist MK $3655^{13}$, did decrease HbAlc, suggesting that sustained, high-level agonism of FGFR1c might be required to improve insulin sensitivity and glycemic control, as indicated by an earlier study comparing weekly administration of efruxifermin with efruxifermin administered every other week in patients with $\mathrm{T} 2 \mathrm{D}^{15}$. Other mechanisms being evaluated as treatments for NASH, such as agonists of farnesoid $\mathrm{X}$ receptors, thyroid hormone receptor- $\beta$ and FGF19, do not improve glycemic control ${ }^{20,30,31}$, emphasizing the unique breadth of efruxifermin's metabolic effects.

Loss of more than $10 \%$ of body weight has been demonstrated to reduce $\mathrm{HFF}$ and to resolve NASH, even for patients with advanced fibrosis $^{32}$, yet many potential NASH therapeutics do not decrease body weight ${ }^{10,31}$, and some are associated with weight gain ${ }^{4}$. Exploratory analyses show that efruxifermin treatment significantly reduced body weight at the highest dose, with a numerical reduction seen at $50 \mathrm{mg}$. Loss of $3.3 \mathrm{~kg}$ over 16 weeks is equivalent to a reduction of $\sim 10 \%$ in daily dietary caloric intake ${ }^{33}$. The weight loss did not appear to result from reduced food intake, as more participants reported increased rather than decreased appetite. Preclinical studies indicate that FGF21 activates brown fat thermogenesis and increases energy expenditure ${ }^{6}$. However, absence of reported hydrosis in this study suggests that any possible thermogenic effect of efruxifermin was not excessive. Reducing body weight also augments insulin sensitivity, suggesting a durable improvement in glycemic control with long-term treatment.

A seemingly paradoxical increase in percentage body fat in the 50- and 70-mg groups is consistent with the association of a common single-nucleotide polymorphism (SNP) in the FGF21 gene with lower body fat in humans but increased waist circumference ${ }^{34}$. The SNP is considered likely to be associated with lower FGF21 function. Thus, efruxifermin might redistribute fat from the viscera to the peripheral tissues, consistent with enhanced insulin sensitivity.

Efruxifermin was generally well tolerated. The most frequently reported AEs were gastrointestinal, mainly mild and transient in nature, reflecting previous observations with efruxifermin ${ }^{15}$ and other FGF21 analogs ${ }^{8,10,11}$. Taking into account the dose-dependent improvements in liver health and exploratory whole-body metabolic endpoints, 28-mg and 50-mg efruxifermin appear to bracket the dose range that combines efficacy with acceptable safety and tolerability. Despite the high incidence of de novo antibody formation, ADA titers were generally low. Few patients developed antibodies that cross-reacted with native FGF21, and only one patient developed neutralizing ADAs.

Strengths of this study include non-invasive measures of liver steatosis and fibrogenesis (MRI-PDFF, markers of liver injury, pro-C3 and ELF), corroborating the observed improvements in liver histopathology. This association offers scope to monitor therapeutic effect non-invasively to confirm that patients are responding to efruxifermin in a way that would be expected to reduce or resolve $\mathrm{NASH}$ pathology during long-term treatment. The study's biopsy procedure was closely defined, yielding specimens of high quality with no inadequate samples.

Limitations of this study include absence of a placebo control for the biopsy-eligible cohort of 50 participants. Only two placebo participants were eligible because the remainder were non-responders-that is, did not achieve $\geq 30 \%$ reduction in HFF. This study was constrained by the COVID-19 pandemic, resulting in loss of $16 \%$ of end-of-study biopsies, and some end-of-study assessments (liver MRI-PDFF and biopsy) were brought forward, resulting in a wider range of assessment periods. Calorie intake and energy expenditure were not controlled, and daily activity was not measured. A third of participants were fibrosis stage F1 and, thus, more susceptible to spontaneous resolution of NASH. To minimize any potential effect on histological outcomes arising from uneven distribution of F1 across treatment groups, patients were stratified by fibrosis stage (F1 versus F2-3) at baseline before random assignment. Comparing the magnitude of HFF reduction in F1 versus F2F3 subgroups suggests that efruxifermin's effects were similar.

In conclusion, efruxifermin significantly reduced liver fat and was generally safe and well tolerated in patients with NASH and F1-F3 fibrosis. The sustained reduction of HFF and liver injury markers after 16 weeks of treatment, combined with exploratory histological and metabolic improvements, suggest that efruxifermin might have the potential to modify the course of NASH progression. Longer-duration studies in larger patient groups are warranted to further evaluate the potential of efruxifermin as a first-line treatment for NASH.

\section{Online content}

Any methods, additional references, Nature Research reporting summaries, source data, extended data, supplementary information, acknowledgements, peer review information; details of author contributions and competing interests; and statements of data and code availability are available at https://doi.org/10.1038/ s41591-021-01425-3.

Received: 11 December 2020; Accepted: 2 June 2021; Published online: 8 July 2021

\section{References}

1. Chalasani, N. et al. The diagnosis and management of nonalcoholic fatty liver disease: practice guidance from the American Association for the Study of Liver Diseases. Hepatology 67, 328-357 (2018).

2. Williams, C. D. et al. Prevalence of nonalcoholic fatty liver disease and nonalcoholic steatohepatitis among a largely middle-aged population utilizing ultrasound and liver biopsy: a prospective study. Gastroenterology 140, 124-131 (2011)

3. Younossi, Z. M. et al. Global epidemiology of nonalcoholic fatty liver disease-meta-analytic assessment of prevalence, incidence, and outcomes. Hepatology 64, 73-84 (2016)

4. Neuschwander-Tetri, B. A. Therapeutic landscape for NAFLD in 2020. Gastroenterology 158, 1984-1998 (2020).

5. Tillman, E. J. \& Rolph, T. FGF21: an emerging therapeutic target for non-alcoholic steatohepatitis and related metabolic diseases. Front. Endocrinol. (Lausanne) 11, 601290 (2020).

6. Geng, L., Lam, K. S. L. \& Xu, A. The therapeutic potential of FGF21 in metabolic diseases: from bench to clinic. Nat. Rev. Endocrinol. 16, 654-667 (2020)

7. Gaich, G. et al. The effects of LY2405319, an FGF21 analog, in obese human subjects with type 2 diabetes. Cell Metab. 18, 333-340 (2013).

8. Talukdar, S. et al. A long-acting FGF21 molecule, PF-05231023, decreases body weight and improves lipid profile in non-human primates and type 2 diabetic subjects. Cell Metab. 23, 427-440 (2016).

9. Charles, E. D. et al. Pegbelfermin (BMS-986036), PEGylated FGF21, in patients with obesity and type 2 diabetes: results from a randomized phase 2 study. Obesity (Silver Spring) 27, 41-49 (2019). 
10. Sanyal, A. et al. Pegbelfermin (BMS-986036), a PEGylated fibroblast growth factor 21 analogue, in patients with non-alcoholic steatohepatitis: a randomised, double-blind, placebo-controlled, phase 2a trial. Lancet 392, 2705-2717 (2019).

11. Kunder, R. et al. Multiple Doses of an anti-FGFR1/KLB bispecific antibody (BFKB8488A) are associated with a decrease in hepatic fat in patients in NAFLD. American Association for the Study of Liver Diseases (AASLD): The Liver Meeting, LP-8 (2019).

12. Wong, C. et al. A bispecific antibody to FGFR1/KLB, BFKB8488A, improves lipid profile, markers of fibrogenesis and liver health in patients with type 2 diabetes-preliminary results from a phase 1b study. American Association for the Study of Liver Diseases (AASLD): The Liver Meeting, P-2310 (2019).

13. DePaoli, A. et al. NGM313, a novel activator of b-klotho/FGFR1c, improves insulin resistance and reduces hepatic fat in obese, nondiabetic subjects. American Diabetes Association https://diabetes.diabetesjournals.org/ content/68/Supplement 1/140-LB (2019).

14. Stanislaus, S. et al. A novel Fc-FGF21 with improved resistance to proteolysis, increased affinity toward $\beta$-klotho, and enhanced efficacy in mice and cynomolgus monkeys. Endocrinology 158, 1314-1327 (2017).

15. Kaufman, A., Abuqayyas, L., Denney, W. S., Tillman, E. J. \& Rolph, T. AKR-001, an Fc-FGF21 analog, showed sustained pharmacodynamic effects on insulin sensitivity and lipid metabolism in type 2 diabetes patients. Cell Rep. Med. 1, 100057 (2020).

16. Lambert, J. E., Ramos-Roman, M. A., Browning, J. D. \& Parks, E. J. Increased de novo lipogenesis is a distinct characteristic of individuals with nonalcoholic fatty liver disease. Gastroenterology 146, 726-735 (2014).

17. Gallego-Escuredo, J. M. et al. Opposite alterations in FGF21 and FGF19 levels and disturbed expression of the receptor machinery for endocrine FGFs in obese patients. Int. J. Obes. (Lond.) 39, 121-129 (2015).

18. $\mathrm{Xu}, \mathrm{J}$. et al. Fibroblast growth factor 21 reverses hepatic steatosis, increases energy expenditure, and improves insulin sensitivity in diet-induced obese mice. Diabetes 58, 250-259 (2009).

19. Harrison, S. A. et al. Efficacy and safety of aldafermin, an engineered FGF19 analog, in a randomized, double-blind, placebo-controlled trial of patients with nonalcoholic steatohepatitis. Gastroenterology 160, 219-231 (2020).

20. Harrison, S. A. et al. NGM282 for treatment of non-alcoholic steatohepatitis: a multicentre, randomised, double-blind, placebo-controlled, phase 2 trial Lancet 391, 1174-1185 (2018).

21. Patel, J. et al. Association of noninvasive quantitative decline in liver fat content on MRI with histologic response in nonalcoholic steatohepatitis. Ther. Adv. Gastroenterol. 9, 692-701 (2016).
22. Harrison, S. A. et al. NGM282 improves liver fibrosis and histology in 12 weeks in patients with nonalcoholic steatohepatitis. Hepatology 5, 303 (2019).

23. Brunt, E. M. et al. Improvements in histologic features and diagnosis associated with improvement in fibrosis in nonalcoholic steatohepatitis: results from the nonalcoholic steatohepatitis clinical research network treatment trials. Hepatology 70, 522-531 (2019).

24. Lanaspa, M. A. et al. Uric acid induces hepatic steatosis by generation of mitochondrial oxidative stress. J. Biol. Chem. 287, 40732-40744 (2012).

25. Afzali, A., Weiss, N. S., Boyko, E. J. \& Ioannou, G. N. Association between serum uric acid level and chronic liver disease in the United States. Hepatology 52, 578-589 (2010).

26. Nielsen, M. J. et al. Markers of collagen remodeling detect clinically significant fibrosis in chronic hepatitis c patients. PLoS ONE 10, e0137302 (2015)

27. Shafiei, M. S., Shetty, S., Scherer, P. E. \& Rockey, D. C. Adiponectin regulation of stellate cell activation via PPAR $\gamma$-dependent and -independent mechanisms. Am. J. Pathol. 178, 2690-2699 (2011).

28. Irvine, K. M. et al. The enhanced liver fibrosis score is associated with clinical outcomes and disease progression in patients with chronic liver disease. Liver Int. 36, 370-377 (2016).

29. Bayly, G. R. in Clinical Biochemistry (eds. Marshall, W. J., Lapsley, M., Day, A. P. \& Ayling, R. M.) 702-736 (Elsevier, 2014).

30. Younossi, Z. M. et al. Obeticholic acid for the treatment of non-alcoholic steatohepatitis: interim analysis from a multicentre, randomised, placebo-controlled phase 3 trial. Lancet 394, 2184-2196 (2019).

31. Harrison, S. A. et al. Resmetirom (MGL-3196) for the treatment of non-alcoholic steatohepatitis: a multicentre, randomised, double-blind, placebo-controlled, phase 2 trial. Lancet 394, 2012-2024 (2019).

32. Vilar-Gomez, E. et al. Weight loss through lifestyle modification significantly reduces features of nonalcoholic steatohepatitis. Gastroenterology 149, 367-378; quiz e14-e15 (2015)

33. Hall, K. D. What is the required energy deficit per unit weight loss? Int. J. Obes. (Lond.) 32, 573-576 (2008).

34. Frayling, T. M. et al. A common allele in FGF21 associated with sugar intake is associated with body shape, lower total body-fat percentage, and higher blood pressure. Cell Rep. 23, 327-336 (2018).

Publisher's note Springer Nature remains neutral with regard to jurisdictional claims in published maps and institutional affiliations.

(c) The Author(s), under exclusive licence to Springer Nature America, Inc. 2021 


\section{Methods}

Study design and participants. This multicenter, randomized, double-blind, placebo-controlled, parallel-group, phase 2a study screened patients at 27 gastroenterology and hepatology clinics in the United States. An expansion of this study in an additional cohort of 30 patients with NASH with compensated cirrhosis is ongoing. This article is focused on the main study, in which eligible patients were adults with biopsy-proven NASH, fibrosis stage $1-3$, NAS of $\geq 4$ (with at least a score of 1 in each of steatosis, ballooning degeneration and lobular inflammation $)^{35}$, HFF of $\geq 10 \%$ by MRI-PDFF and FibroScan measurement $>7.0 \mathrm{kPa}$. Target enrollment in the main study was 80 patients. Excluded were patients with fibrosis stage 4 (cirrhosis), history of decompensated liver disease, liver transplantation, hepatocellular carcinoma or chronic hepatitis B or C. All inclusion and exclusion criteria are provided in the supplementary information (Supplementary Table 7). All participants provided written informed consent before enrollment. This study accorded with the ethical principles of the Declaration of Helsinki and was consistent with the International Conference on Harmonization of Good Clinical Practice and applicable regulatory requirements. A central institutional review board (Advarra Institutional Review Board, 00000971) approved the study protocol and all amendments.

Randomization and masking. The principal investigators enrolled participants. Investigators, radiologists, pathologist, staff, patients, sponsor and medical monitor remained masked to treatment groups during the study. Efruxifermin $\left(70 \mathrm{mg} \mathrm{ml}^{-1}\right)$ and placebo, identical in appearance, were provided in 6-ml sterile vials containing $1.1 \mathrm{ml}$. Diluent vials were provided for preparation of the 28 - and 50-mg doses. At each site, doses were prepared in pre-filled syringes labeled with unique code numbers by an unblinded pharmacist not otherwise involved with the study. Patients were stratified by baseline HFF ( $<15 \%$ versus $\geq 15 \%)$ and fibrosis stage (F1 versus F2-F3), with site effect considered, and then randomly assigned to one of four treatment groups in a 1:1:1:1 ratio (efruxifermin $28 \mathrm{mg}$, efruxifermin $50 \mathrm{mg}$, efruxifermin $70 \mathrm{mg}$ or placebo) via the centrally administered Medpace ClinTrak Interactive Response Technology system using a dynamic allocation method. A minimization algorithm was used to calculate a score to determine the optimal treatment group assignment.

Procedures. Study drug $(1 \mathrm{ml})$ was administered subcutaneously into the abdomen weekly for 16 weeks. Standard protocols were followed for injection site evaluation. Enrolled patients participated in the study for $\sim 30$ weeks: up to 8 weeks for screening, 16 weeks on-treatment, 4 weeks of safety follow-up (week 20) and up to 4 weeks for a post-treatment liver biopsy and MRI-PDFF in eligible participants (see Extended Data Fig. 1 for study design). MRI-PDFF was measured at baseline, week 6 , week 12 and, for responders (those with $\geq 30 \%$ relative HFF reduction at week 12), between weeks 20 and 24 . Historical (within $180 \mathrm{~d}$ of randomization) or new liver biopsies were obtained for screening and pre-baseline evaluation of histopathology. Tissue was processed at a central laboratory (Pacific Rim Pathology Laboratory) using routine methods. Sections of 3-4 $\mu \mathrm{m}$ were stained with trichrome and hematoxylin and eosin. NAS grading of steatosis, ballooning and lobular inflammation ${ }^{35}$ and staging of fibrosis were performed by a single pathologist. End-of-treatment biopsies were independently assessed in the same blinded manner. MRI scans were read centrally. Due to the COVID-19 pandemic, the window for repeat biopsy was expanded to allow earlier completion. Last visit assessments were permitted as early as week 16 . The median time for post-treatment biopsy among the BAS was week 20 .

Bone mineral density and body fat distribution were assessed by dual-energy X-ray absorptiometry at screening and week 16. Vital signs, 12-lead electrocardiogram, clinical laboratory testing (hematology, chemistry and urinalysis), lipid parameters and other biomarkers were assessed at least every 4 weeks. Safety and tolerability were assessed at all time points and during follow-up.

Outcomes. The primary endpoint was absolute change from baseline in HFF at week 12 in all randomized patients (FAS). The secondary objectives were to evaluate: the percent change from baseline in HFF at week 12; the proportion of responders, defined as patients who achieved a relative reduction of $\geq 30 \%$ in HFF at week 12 (MRI-PDFF responders); the proportion of NAS responders-that is, patients who had a decrease of $\geq 2$ points in NAS with at least a 1-point reduction in either lobular inflammation or hepatocellular ballooning and no concurrent worsening of fibrosis stage; and the change from baseline in ALT at week 12; and to assess the safety and tolerability of efruxifermin in patients with NASH.

Key exploratory endpoints included change from baseline in markers of liver injury and function, histology endpoints and non-invasive markers of fibrosis, markers of glycemic control, lipoprotein profiles and body weight. A full list of exploratory endpoints is provided in the supplementary information (Supplementary Table 8).

Statistical analysis. As an exploratory study, no formal power calculations were conducted to determine sample size. Target participant numbers $(n=20$ per treatment group) were based on clinical experience with other similar proof-of-concept studies ${ }^{10}$. With a $5 \%$ significance level, 20 participants receiving placebo and an s.d. estimated as $5.2 \%$, the power for $n=20$ participants with active treatment is $\sim 91 \%$ to detect a true, baseline-adjusted, mean absolute decrease of $5 \%$ from baseline in MRI-PDFF-estimated hepatic fat compared to placebo, using a one-sided $t$-test for decrease from placebo.

For primary and secondary continuous endpoints related to HFF, an analysis of covariance (ANCOVA) model was used, with treatment group and fibrosis stage (F1 versus F2-F3) as factors and baseline HFF as a covariate. LS means, one-sided $97.5 \%$ CIs and one-sided $P$ values were calculated for the primary endpoint. Normality was tested for the model residuals by the Shapiro-Wilk test. A step-down fixed hypothesis testing sequence was used to control for type I error rate inflation caused by multiplicity issues. The next lower dose was compared only if the higher dose was significantly better than placebo. Multiple imputation was employed for missing primary efficacy values in the FAS. For continuous secondary efficacy endpoints, LS means, $95 \%$ CIs and $P$ values were calculated for absolute and relative change. Fixed hypothesis testing sequence was used to control the multiplicity for each active dose compared to placebo. Analyses were conducted on the FAS and appropriate data subsets.

Three secondary endpoint responder analyses were performed. MRIPDFF responders in the FAS were calculated with missing values imputed as non-responders and in the MRI-PDFF (HFF) evaluable analysis set (MAS, the subgroup with baseline and week 12 MRI-PDFF assessments) without imputation. NAS responders in the liver biopsy evaluable analysis set (BAS, MRI-PDFF responders with liver biopsy at baseline and after treatment) were calculated without imputation.

ALT and continuous exploratory endpoints were analyzed by treatment using an ANCOVA model or post hoc by mixed model repeated measures. All dose groups were tested for difference from placebo in the post hoc analysis and on exploratory endpoints. Exploratory responder analyses were performed on the appropriate analysis set. SAS version 9.4 was used for all statistical analyses.

The safety analysis set comprised all patients who received at least one dose of the study drug. An external data monitoring committee consisting of two hepatologists, one cardiologist and a statistician oversaw the study. The trial was registered with ClinicalTrials.gov (NCT03976401).

Role of the funding source. The study was funded by Akero Therapeutics, which, in collaboration with the authors, was involved in study design and data collection. The authors (some of whom are Akero Therapeutics employees) were responsible for analysis and interpretation of data and writing the report. All authors had full access to all study data, and the corresponding author had final responsibility for the decision to submit for publication.

Reporting Summary. Further information on research design is available in the Nature Research Reporting Summary linked to this article.

\section{Data availability}

The datasets generated and/or analyzed in this study are considered commercially sensitive and, therefore, are not publicly available. Requests for data supporting findings in the manuscript should be made to the corresponding author and will be reviewed individually. Data might be shared in the form of aggregate data summaries and via a data transfer agreement. Individual participant-level raw data containing confidential or identifiable patient information are subject to patient privacy and cannot be shared.

\section{References}

35. Kleiner, D. E. et al. Design and validation of a histological scoring system for nonalcoholic fatty liver disease. Hepatology 41, 1313-1321 (2005).

\section{Acknowledgements}

The authors thank the patients and their families. We also thank afPharmative Insights for medical writing support and R. Shringarpure for critical review of the manuscript. The study was funded by Akero Therapeutics.

\section{Author contributions}

All authors were involved in data interpretation and review and writing of the manuscript. A.C., B.D.T., K.Y., S.A.H. and T.P.R. designed the study. B.L.F., C.A.B., G.N., P.J.R., R.P. and S.A.H. collected the data. C.A.B., C.H., E.F., E.J.T., T.P.R. and S.A.H. analyzed the data.

\section{Competing interests}

B.L.F., G.N., P.J.R., R.P. and S.A.H. received research funding from Akero Therapeutics as part of the current study. C.A.B. and C.H. are employees of research partners who received payment from Akero Therapeutics for services as part of the current study. A.C., B.D.T., E.F., E.J.T., K.Y. and T.P.R. are employees of Akero Therapeutics. A.C., B.D.T., E.F., E.J.T., K.Y., S.A.H. and T.P.R. own stock and/or stock options of Akero Therapeutics. 


\section{Additional information}

Extended data is available for this paper at https://doi.org/10.1038/s41591-021-01425-3.

Supplementary information The online version contains supplementary material available at https://doi.org/10.1038/s41591-021-01425-3.

Correspondence and requests for materials should be addressed to K.Y.
Peer review information Nature Medicine thanks Arun Sanyal, Martin Wagner and the other, anonymous, reviewer(s) for their contribution to the peer review of this work. Jennifer Sargent was the primary editor on this article and managed its editorial process and peer review in collaboration with the rest of the editorial team.

Reprints and permissions information is available at www.nature.com/reprints. 


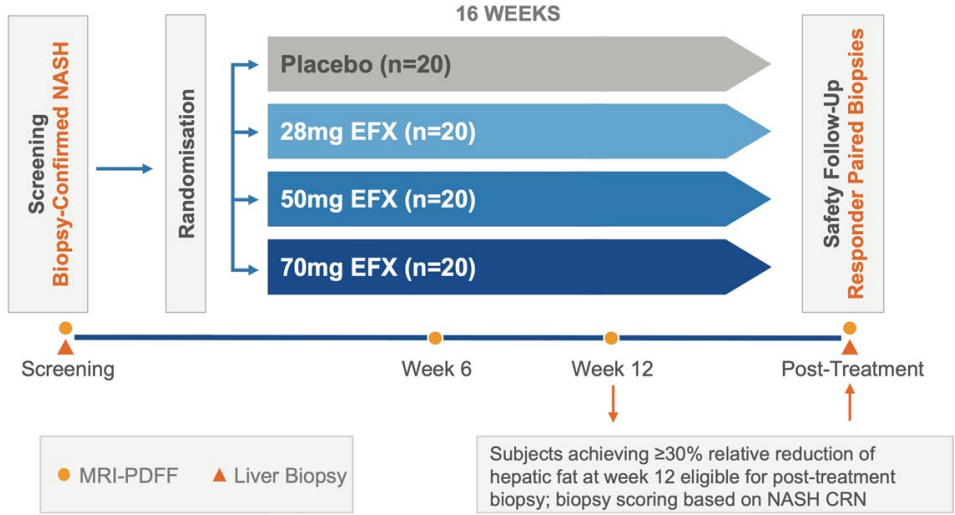

Extended Data Fig. 1 | Summary of study design. MRI-PDFF, magnetic resonance imaging-proton density fat fraction; NASH, non-alcoholic steatohepatitis; NASH CRN, NASH Clinical Research Network. 


\section{Reporting Summary}

Nature Research wishes to improve the reproducibility of the work that we publish. This form provides structure for consistency and transparency in reporting. For further information on Nature Research policies, see our Editorial Policies and the Editorial Policy Checklist.

\section{Statistics}

For all statistical analyses, confirm that the following items are present in the figure legend, table legend, main text, or Methods section.

n/a Confirmed

$\bigotimes$ The exact sample size $(n)$ for each experimental group/condition, given as a discrete number and unit of measurement

\ A statement on whether measurements were taken from distinct samples or whether the same sample was measured repeatedly

The statistical test(s) used AND whether they are one- or two-sided

Only common tests should be described solely by name; describe more complex techniques in the Methods section.

Х A description of all covariates tested

\A description of any assumptions or corrections, such as tests of normality and adjustment for multiple comparisons

$\triangle$ A full description of the statistical parameters including central tendency (e.g. means) or other basic estimates (e.g. regression coefficient) AND variation (e.g. standard deviation) or associated estimates of uncertainty (e.g. confidence intervals)

For null hypothesis testing, the test statistic (e.g. $F, t, r$ ) with confidence intervals, effect sizes, degrees of freedom and $P$ value noted Give $P$ values as exact values whenever suitable.

Х $\square$ For Bayesian analysis, information on the choice of priors and Markov chain Monte Carlo settings

$\bigotimes \square$ For hierarchical and complex designs, identification of the appropriate level for tests and full reporting of outcomes

$\bigotimes \square$ Estimates of effect sizes (e.g. Cohen's $d$, Pearson's $r$ ), indicating how they were calculated

\section{Our web collection on statistics for biologists contains articles on many of the points above.}

\section{Software and code}

Policy information about availability of computer code

Data collection Patients were randomized via the centrally-administered Medpace ClinTrak Interactive Response Tehcnology System using a dynamic allocation method. A minimization algorithm was used to calculate a score to determine the optimal treatment group assignment.

Data analysis SAS version 9.4 was used in the analysis of data in this study

For manuscripts utilizing custom algorithms or software that are central to the research but not yet described in published literature, software must be made available to editors and

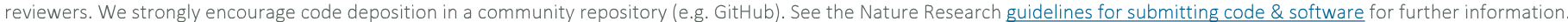

\section{Data}

Policy information about availability of data

All manuscripts must include a data availability statement. This statement should provide the following information, where applicable:

- Accession codes, unique identifiers, or web links for publicly available datasets

- A list of figures that have associated raw data

- A description of any restrictions on data availability

The datasets generated and/or analyzed during the current study are considered commercially sensitive and therefore are not publicly available. Requests for data supporting findings in the manuscript should be made to the corresponding author and will be reviewed individually. Data may be shared in the form of aggregate data summaries and via a data transfer agreement. Individual participant level raw data containing confidential or identifiable patient information is subject to patient privacy and cannot be shared. 
Please select the one below that is the best fit for your research. If you are not sure, read the appropriate sections before making your selection.

\ Life sciences

Behavioural \& social sciences

Ecological, evolutionary \& environmental sciences

For a reference copy of the document with all sections, see nature.com/documents/nr-reporting-summary-flat.pdf

\section{Life sciences study design}

All studies must disclose on these points even when the disclosure is negative.

Sample size No a priori formal power calculations were conducted to determine sample size. Subject numbers were chosen based on clinical experience with other similar proof of concept studies that have demonstrated significant reduction in liver fat content (Sanyal A et al. Lancet.

2018;392(10165):2705-2717). With a 5\% significance level, 20 subjects receiving placebo, and a standard deviation estimated as 5.2\%, the power for $n=20$ subjects with active treatment is $~ 91 \%$ to detect a true, baseline-adjusted, mean decrease of $5 \%$ from baseline in MRI-PDFFestimated hepatic fat compared to placebo, using a one-sided t-test for decrease from placebo.

Data exclusions No data were excluded from analyses

Replication The article describes a clinical trial with a proprietary agent and replication by other parties is currently not applicable

Randomization Patients were stratified by baseline hepatic fat fraction ( $<15 \%$ versus $<=15 \%$ ) and fibrosis stage (F1 versus $F 2-F 3$ ), then randomly assigned to treatment arm using a centrally-administered interactive response technology.

Blinding Investigators, radiologists, pathologist, staff, patients, sponsor, and medical monitor remained blinded to treatment groups during the study

\section{Reporting for specific materials, systems and methods}

We require information from authors about some types of materials, experimental systems and methods used in many studies. Here, indicate whether each material, system or method listed is relevant to your study. If you are not sure if a list item applies to your research, read the appropriate section before selecting a response.

\begin{tabular}{l|l} 
Materials \& experimental systems \\
\hline $\mathrm{n} / \mathrm{a}$ & Involved in the study \\
\hline & $\square$ Antibodies \\
\hline & $\square$ Eukaryotic cell lines \\
$\square$ & $\square$ Palaeontology and archaeology \\
$\square$ & $\square$ Animals and other organisms \\
$\square$ & $\square$ Human research participants \\
$\square$ & $\square$ Clinical data \\
$\square$ & $\square$ Dual use research of concern
\end{tabular}

\begin{tabular}{l|l} 
Methods \\
\hline n/a & Involved in the study \\
$\searrow$ & $\square$ ChIP-seq \\
$\searrow$ & $\square$ Flow cytometry \\
$\searrow$ & $\square$ MRI-based neuroimaging
\end{tabular}

\section{Human research participants}

\section{Policy information about studies involving human research participants}

Population characteristics

Participants were approximately 52 years of age on average, and $58 \%$ were female. The majority of participants were of White Race and half were of hispanic ethnicity. Baseline demographics and disease characteristics were similar across treatment groups. Fibrosis stages (F1 [36\%], F2 [33\%], or F3 [31\%]) were evenly distributed across treatment groups. Hepatic fat fraction was similar in all treatment groups (19.3\%, $21 \cdot 4 \%, 18 \cdot 3 \%$ and $19 \cdot 4 \%)$.

Recruitment

Standard site outreach and physician referrals. The study design ensured minimization of any bias. The study was a randomized double blind study where both participants, clinical investigators and site personnel as well as sponsor teams were blinded to treatment assignment precluding any opportunity for self-selection bias.

Ethics oversight

Advarra Institutional Review Board

Note that full information on the approval of the study protocol must also be provided in the manuscript. 
Policy information about clinical studies

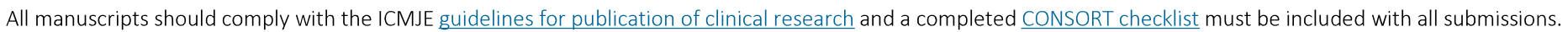

Clinical trial registration

Study protocol

Data collection

Outcomes
ClinicalTrials.gov, NCT03976401

Study protocol not publicly available

Between May 28 and November 15, 2019, 356 individuals were screened at 27 US gastroenterology and hepatology clinics, with 80 patients from 24 sites enrolled between July 2 and December 16, 2019. Data collection occurred between May 282019 and May 04 2020. The database was locked on Aug 262020

The primary endpoint was absolute change from baseline in HFF at week 12 in all randomized patients (full analysis set [FAS]). The secondary objectives were to evaluate the percent change from baseline in HFF at week 12; the proportion of responders, defined as patients who achieved a relative reduction of $\geq 30 \%$ in HFF at week 12 (MRI-PDFF responders); the proportion of NAS responders, i.e., patients who had a decrease of $>$ or $=2$ points in NAS with at least a 1-point reduction in either lobular inflammation or hepatocellular ballooning and no concurrent worsening of fibrosis stage; the change from baseline in alanine aminotransferase (ALT) at week 12; and to assess the safety and tolerability of efruxifermin in patients with NASH. For primary and secondary continuous endpoints related to HFF, an analysis of covariance (ANCOVA) model was used with treatment group and fibrosis stage (F1 versus F2F3) as factors and baseline HFF as a covariate. Least squares (LS) means, 1-sided 97.5\% Cl, and 1-sided p-values were calculated for the primary endpoint. Normality was tested for the model residuals by the Shapiro-Wilk test. A step-down fixed hypothesis testing sequence was used to control for Type I error rate inflation caused by multiplicity issues. The next lower dose was compared only if the higher dose was significantly better than placebo. Multiple imputation was employed for missing primary efficacy values in the FAS. For continuous secondary efficacy endpoints, LS means, 95\% Cls, and p-values were calculated for absolute and relative change. Fixed hypothesis testing sequence was used to control the multiplicity for each active dose compared with placebo. Analyses were conducted on the FAS and appropriate data subsets.

Three secondary endpoint responder analyses were performed. MRI-PDFF responders in the FAS were calculated with missing values imputed as non-responders, and in the MRI-PDFF (HFF) evaluable analysis set (MAS, the subgroup with baseline and week 12 MRIPDFF assessments) without imputation. NAS responders in the liver biopsy evaluable analysis set (BAS, MRI-PDFF responders with liver biopsy at baseline and post treatment) were calculated without imputation. Additional details are defined in the Study Protocol, and methods of assessment are detailed in the Statistical Analysis Plan. 